Original Contribution

\title{
Diseases associated with electrolyte imbalance in the ED: age-related differences
}

\author{
Mauro Giordano, MD ${ }^{\mathrm{a}, *}$, Tiziana Ciarambino, MD ${ }^{\mathrm{a}}$, Pietro Castellino, MD ${ }^{\mathrm{b}}$, Lorenzo Malatino, $\mathrm{MD}^{\mathrm{b}}$, \\ Salvatore Di Somma, MD ${ }^{\mathrm{c}}$, Gianni Biolo, MD ${ }^{\mathrm{d}}$, Giuseppe Paolisso, MD ${ }^{\mathrm{a}}$, Luigi Elio Adinolfi, MD ${ }^{\mathrm{a}}$ \\ a Department of Medical, Surgical, Neurological, Metabolic and Geriatrics Sciences, Second University of Naples, Naples, Italy \\ ${ }^{\mathrm{b}}$ Department of Internal Medicine of Catania, Catania, Italy \\ c Department of Medical-Surgery Sciences and Translational Medicine, University La Sapienza Rome, Sant'Andrea Hospital, Rome, Italy \\ d Department of Medical Sciences, University of Trieste, Trieste, Italy
}

\section{A R T I C L E I N F O}

\section{Article history:}

Received 5 February 2016

Received in revised form 18 April 2016

Accepted 18 May 2016

\begin{abstract}
A B S T R A C T
Objective: The objective of the study is to investigate the prevalence of electrolyte imbalance (EI) in the emergency department (ED) with systemic diseases in different decades of life.

Methods: We enrolled patients admitted to the ED. The population study included 7941 patients, subdivided in 3 groups: young group (Y), middle-aged group (MA), and elderly group (E).

Results: We observed EI in $13.7 \%$ of the whole population. Hyponatremia $\left(\mathrm{hNa}^{+}\right)$is the most frequent EI (44\%) followed by hypokalemia $\left(\mathrm{hK}^{+}\right)(39 \%)$, hyperkalemia $\left(\mathrm{HK}^{+}\right)(13 \%)$, and hypernatremia $\left(\mathrm{HNa}^{+}\right)(4.4 \%)$. In the Y group, the EI occurred in $7.1 \%$ of all patients $(P<.05$ vs MA and $\mathrm{E}$ ), whereas in the MA group, they were shown in $11.5 \%$ of patients and in the E group in $22 \%$ of all patients group $(P<.05$ vs MA and $Y)$. In the $Y$ group, gastrointestinal diseases are the most frequently associated disease (24.6\%; $P<.05$ vs MA and $E)$. In the MA group, the most frequently associated disease was a current cardiovascular disease (29.7\%; $P<.05$ vs $Y$ and $\mathrm{E})$. In the E group, the frequently associated diseases are cardiovascular $(22.8 \% ; P<.05$ vs $\mathrm{Y})$ and lung diseases (16.7\%; $P<.05$ vs MA and $\mathrm{Y})$.

Conclusions: In our study, $13.7 \%$ of all patients showed an EI, and only $2 \%$ of cases were alone without any associated systemic disease. Most EIs are associated to other systemic diseases. The present data also depict different age-related and disease-associated prevalence patterns of EI, thus highlighting a complex clinical scenario.
\end{abstract}

(c) 2016 Published by Elsevier Inc.

\section{Introduction}

Electrolyte imbalance (EI) is an independent predictor of mortality, and the prevalence and incidence of EI in the emergency department (ED) are of great clinical importance because these disorders have been associated with an increased risk of mortality [1-3]. Thus, prompt diagnosis and treatment are crucial in the management of patients admitted to the ED [4]. Although dysnatremias and dyskalemias are commonly shown in hospitalized patients [5], little is so far known data on the prevalence in the ED [6]. Recently, age-related differences in EI have been reported in the ED [5]. In addition, EI in the ED is often associated with systemic diseases [7-8].

\footnotetext{
* Corresponding author at: Department of Medical, Surgical, Neurological, Metabolic and Geriatrics Sciences, Second University of Naples, Naples, Italy.

E-mail address: mauro.giordano@unina2.it (M. Giordano).
}

To our knowledge, the association between EI with systemic disease in the ED has yet to be reported. Thus, we aimed to investigate the prevalence of dysnatremias and dyskalemias in ED patients and their association to major systemic diseases in different age groups.

\section{Methods}

We carried out an observational study by recruiting all patients admitted to the ED of the Marcianise Hospital, at the Second University of Naples, between January 1, 2014, and December 31, 2014, with assessment of EI upon admission. The study design is a cross-sectional retrospective chart review. Investigators trained in the systematic chart review manually reviewed all charts meeting inclusion criteria. Patients with incomplete charts were excluded. The abstracted data were stored using an electronic spreadsheet on password-protected computers. The study population included 7941 patients. It was subdivided into 3 groups: the young group $(\mathrm{Y})$, consisting of 2358 patients aged between 18 and 40 years; the middle-aged group (MA) consisting of 2975 patients aged between 41 and 65 years; and the elderly group (E), 
including 2608 patients older than 65 years. Demographic data were obtained from all patients, and final diagnosis was derived from the International Classification of Diseases, 10th Revision. Blood samples were collected within 5 minutes after patient's arrival in the ED. We used baseline characteristics and serum creatinine to calculate the estimated glomerular filtration rate in accordance with the Chronic Kidney Disease Epidemiology formula [9]. The blood sample from each patient was divided into several tubes or syringes for immediate analysis. The blood samples in BD Vacutainer Blood Collection Tubes (Becton, Dickinson and Company, Franklin Lakes, NJ) containing lithium heparin were cooled in ice-cold water before analysis for their ammonia levels. The blood samples in BD Vacutainer Blood Collection Tubes containing plasma separating tube gels and lithium heparin were sent to the clinical laboratory for other biochemistry analyses. Biochemical data were measured using a Vitros Fusion 5,1 FS Automated Chemistry Analyzer (Ortho Clinical Diagnostics, Johnson \& Johnson Co, New Brunswick, $\mathrm{NJ}$ ). The analyses included serum creatinine, glucose, aspartate aminotransferase, alanine aminotransferase, creatine kinase (CK), CK-MB, troponin $\mathrm{T}$, potassium, sodium, calcium, magnesium, chloride, lactate, and ammonia. The blood samples in BD Vacutainer Blood Collection Tubes containing potassium EDTA were immediately sent to the clinical laboratory. Complete blood counts were determined using a COULTER LH 750 Hematology Analyzer (Beckman Coulter Co, Brea, CA) for white blood cells, hemoglobin, and platelets. The blood gas samples in Luer Slip syringes containing heparin were analyzed to obtain $\mathrm{pH}$ values. The blood gas analyses were determined using a Gem Premier 4000 Analyzer (Instrumentation Laboratory Company, Inc, Bedford, MA). Using these data, we calculated the prevalence rates for dyskalemias and dysnatremias defined according to the reference range of our central laboratory. Hypokalemia was defined as a serum potassium level less than $3.5 \mathrm{mmol} / \mathrm{mol}$; and hyperkalemia, as a serum potassium level exceeding $5.5 \mathrm{mmol} / \mathrm{L}$. Hyponatremia was defined as a serum sodium level less than $135 \mathrm{mmol} / \mathrm{L}$; and hypernatremia, as a serum sodium exceeding $145 \mathrm{mmol} / \mathrm{L}$. In addition, we gathered laboratory data on serum electrolytes, creatinine, and venous blood gas analyses of all patients presenting with EI. Patients with a known pregnancy and severe hypothermia (defined as a body temperature body $30^{\circ} \mathrm{C}$ ) as well as patients who were younger than 18 years and, finally, surgical patients were excluded from the study. The experimental protocol was reviewed and approved by the Ethics Committee of the Second University of Naples. Voluntary written consent was obtained before their participation. The study was carried out according to the Helsinki declaration.

\section{Results}

During the study period (from January 1 to December 31, 2014), a total of 7941 patients presented to the Department of Emergency Medicine, and all received a general evaluation and assessment of blood serum samples.

The mean age of patients was $52.3 \pm 3$ years, and $52 \%$ of patients were male. Patients were divided in 3 age groups: patients aged from 18 to 40 , Y group; patients aged from 41 to 65 years, MA group; and patients aged older than 65 years, E group.

The Y group was characterized by 2358 patients with the mean age of $30.3 \pm 2$ years. The MA group was characterized by 2975 patients with the mean age of $52.0 \pm 3$ years. The E group was characterized by 2608 patients with the mean age of $74.8 \pm 2$ years. The characteristics of all patients subdivided for age are reported in Table 1.

Electrolyte imbalance was present in 1087 (13.7\%) of the cohort. In particular, hyponatremia $\left(\mathrm{hNa}^{+}\right)$resulted the most frequent $\mathrm{EI}$ in 468 patients (44\%) followed by hypokalemia $\left(\mathrm{hK}^{+}\right)$in 431 patients $(39 \%)$, hyperkalemia $\left(\mathrm{HK}^{+}\right)$in 141 patients $(13 \%)$, and hypernatremia $\left(\mathrm{HNa}^{+}\right)$in 47 patients (4.4\%).

According to age, the prevalence of EI in the Y group occurred in 167 patients $(7.1 \%)(P<.05$ vs MA and $\mathrm{E})$ but increased in the MA group as
Table 1

Clinical characteristics of study population divided in 3 groups: $\mathrm{Y}$ (18-40 years old), MA (41-65 years old), and E ( $>65$ years old)

\begin{tabular}{lllll}
\hline & All patients & $\mathrm{Y}$ & $\mathrm{MA}$ & $\mathrm{E}$ \\
\hline $\mathrm{n}(\mathrm{M})$ & 7941 & $2358(1174)$ & $2975(1480)$ & $2608(1300)$ \\
$\mathrm{Age}(\mathrm{y})$ & $52.3 \pm 3$ & $30.3 \pm 2$ & $52.0 \pm 3$ & $74.8 \pm 2$ \\
$\mathrm{CrCl}\left(\mathrm{mL} / \mathrm{min} / 1.73 \mathrm{~m}^{2}\right)$ & $88 \pm 2.0$ & $116 \pm 5.0$ & $89 \pm 3.9$ & $59 \pm 2.7^{\mathrm{a}, \mathrm{b}}$ \\
$\mathrm{Cr}(\mathrm{mg} / \mathrm{dL})$ & $1.01 \pm 0.2$ & $0.87 \pm 0.3$ & $0.97 \pm 0.2$ & $1.21 \pm 0.2^{\mathrm{a}, \mathrm{b}}$ \\
$\mathrm{All} \mathrm{EI}(\%)$ & 13.7 & 7.1 & 11.5 & $22.0^{\mathrm{a}, \mathrm{b}}$ \\
$\mathrm{hNa}^{+}(\%)$ & 44.0 & 33.4 & 39.8 & $47.7^{\mathrm{a}, \mathrm{b}}$ \\
$\mathrm{hNa}^{+}($mean) & $116.5 \pm 0.6$ & $120.1 \pm 0.5$ & $117.3 \pm 0.4$ & $112.2 \pm 0.6^{\mathrm{a}, \mathrm{b}}$ \\
$\mathrm{HNa}^{+}(\%)$ & 4.4 & 5.6 & 4.7 & 3.7 \\
$\mathrm{HNa}^{+}($mean $)$ & $147.6 \pm 0.9$ & $149.3 \pm 0.6$ & $147.3 \pm 0.6$ & $146.3 \pm 0.6$ \\
$\mathrm{hK}^{+}(\%)$ & 39.0 & $58.7^{\mathrm{b}, \mathrm{c}}$ & $48.0^{\mathrm{b}}$ & 29.1 \\
$\mathrm{hK}^{+}$(mean) & $3.1 \pm 0.1$ & $2.9 \pm 0.6^{\mathrm{b}, \mathrm{c}}$ & $3.1 \pm 0.4$ & $3.2 \pm 0.5$ \\
$\mathrm{HK}^{+}(\%)$ & 13 & 2.4 & 7.4 & $19.5^{\mathrm{a}, \mathrm{b}}$ \\
$\mathrm{HK}^{+}$(mean) & $5.7 \pm 0.8$ & $5.5 \pm 0.6$ & $5.9 \pm 0.6$ & $6.2 \pm 0.6^{\mathrm{a}, \mathrm{b}}$ \\
\hline
\end{tabular}

Abbreviations: $\mathrm{CrCl}$, creatinine clearance; $\mathrm{Cr}$, serum creatinine.

a $P<.05$ vs $Y$ group.

b $P<.05$ vs MA group.

c $P<.05$ vs E group.

showed in 342 patients (11.5\%) and in the E group as occurred in 578 patients $(22.2 \%)(P<.05$ vs MA and $Y)$.

In particular, in the $Y$ group (2358 patients), the $\mathrm{hK}^{+}$was the most frequent $\mathrm{EI}$ as it was present in 58.7\% (99/167 patients) $(P<.05$ vs MA and $\mathrm{E}$ ), followed by the $\mathrm{hNa}^{+}$showed in $33.4 \%$ (56/167 patients); the $\mathrm{HNa}^{+}$occurred in 5.6\% (9/167 patients), and the $\mathrm{HK}^{+}$was reported in $2.4 \%$ (4/167 patients). In the MA group (2975 patients), the most frequent EI was hK ${ }^{+}$and occurred in $48 \%$ (164/342 patients) $(P<.05$ vs E), followed by the $\mathrm{hNa}^{+}$that occurred in $39.8 \%$ (136/342 patients); the $\mathrm{HK}^{+}$was reported in $7.4 \%$ (25/342 patients), and the $\mathrm{HNa}^{+}$was observed in $4.7 \%$ ( $16 / 342$ patients). In the E group (2608 patients), the most frequent EI was $\mathrm{hNa}^{+}$and occurred in $47.7 \%$ (276/578 patients) $(P<.05$ vs MA and $\mathrm{Y})$, followed by the $\mathrm{hK}^{+}$that occurred in $29.1 \%$ ( $168 / 578$ patients), the $\mathrm{HK}^{+}$that occurred in $19.5 \%$ (112/578 patients), and $\mathrm{HNa}^{+}$that occurred in 3.7\% (21/578 patients) (Fig. 1; Table 1).

The most frequent diseases associated with EI are cardiovascular in 1802 patients (22.7\%), followed by lung in 984 patients (12.4\%) and gastrointestinal (GI) diseases in 1151 patients (14.5\%) (Table 2). We also reported the most frequently associated disease in 3 groups (Table 2). In the Y group, GI disease is the most frequent pathology that occurred in 580 patients $(24.6 \%)(P<.05$ vs MA and $\mathrm{E})$. In the MA group, the most frequently associated disease represented is cardiovascular disease that occurred in 883 patients (29.7\%) $(P<.05$ vs $\mathrm{Y}$ and $\mathrm{E})$. In the E group, the most frequent pathologies were cardiovascular in 594 of the patients $(22.8 \%)(P<.05$ vs $Y)$ and lung diseases in 435 of the patients $(16.7 \%)$ $(P<.05$ vs MA and $Y)$ (Fig. 2$)$.

Hospitalization rate in EI patients greatly varied in the 3 age groups. In fact, in the Y group, it occurred in only 3.6\% (6 of the 167 patients); in the MA group, it increased to $16 \%$ ( 55 of the 342 patients) ( $P<0.01$ vs Y);

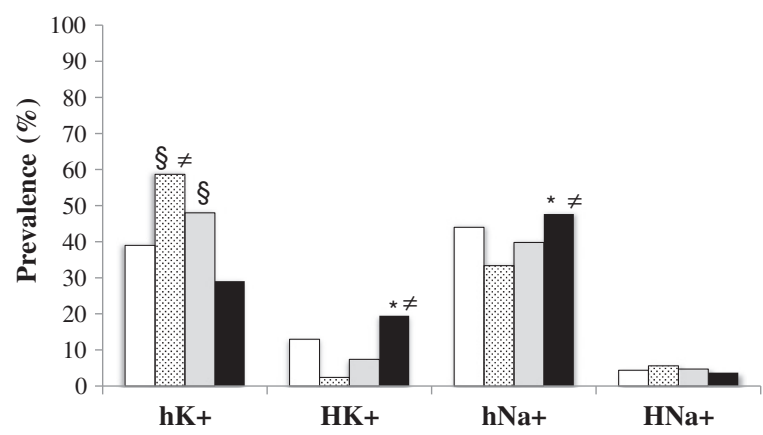

Fig. 1. Hypernatremia (HNa), hyponatremia (hNa), hyperkalemia (HKa), and hypokaliemia (hK) in all patients group (white column), Y group (dotted column), MA group (gray column), and E group (black column). Values are expressed as percentages. ${ }^{*} P<.05$ vs Y group; ${ }^{\neq} P<.05$ vs MA group; ${ }^{\S} P<.05$ vs E group. 
Table 2

Diseases associated with EI on admission to the ED in study population divided in 3 groups: $\mathrm{Y}$ (18-40 years old), MA (41-65 years old), and E ( $>65$ years old)

\begin{tabular}{lllll}
\hline Associated disease & All & Y & MA & E \\
\hline Cardiovascular (\%) & 22.7 & 8.7 & $29.7^{\mathrm{a}, \mathrm{b}}$ & $22.8^{\mathrm{a}}$ \\
Lung (\%) & 12.4 & 4.0 & $9.4^{\mathrm{a}}$ & $16.7^{\mathrm{a}, \mathrm{c}}$ \\
Neurologic (\%) & 9.2 & 13.0 & 9.0 & 8.1 \\
Hepatological (\%) & 5.7 & 0.1 & 5.5 & $7.5^{\mathrm{a}, \mathrm{c}}$ \\
GI (\%) & 14.5 & $24.6^{\mathrm{b}, \mathrm{c}}$ & 11.3 & 7.7 \\
Pain (\%) & 8.1 & $15.9^{\mathrm{b}, \mathrm{c}}$ & 6.6 & 1.9 \\
Psychiatric (\%) & 11.4 & 17.6 & 16.3 & 18.3 \\
Kidney (\%) & 1.0 & 0.1 & 0.8 & $1.6^{\mathrm{a}, \mathrm{c}}$ \\
EI (\%) & 1.6 & 0.8 & 2.0 & $2.3^{\mathrm{a}}$ \\
Immunological (\%) & 0.6 & 0.8 & 0.4 & 0.5 \\
Endocrine (\%) & 3.6 & 2.4 & 3.5 & $4.0^{\mathrm{a}}$ \\
Hematological (\%) & 1.5 & 0.8 & $2.0^{\mathrm{a}}$ & 1.4 \\
Infectious (\%) & 5.1 & $6.3^{\mathrm{c}}$ & 3.5 & 5.6 \\
Other (\%) & 3.3 & 5.6 & 2.0 & 2.3 \\
\hline
\end{tabular}

Values are reported as percentages.

a $P<.05$ vs $Y$ group.

b $P<.05$ vs E group.

c $P<.05$ vs MA group.

and in the E group, it markedly increased to 39\% (225 of the 578 patients) $(P<.01$ vs $\mathrm{Y}$ and MA).

\section{Statistical analyses}

Data analyses were performed using SPSS, version 19.0 (IBM Corp, Armonk, NY). Statistical significance was set at $P<.05$ for all procedures. Analyses of variance or $t$ tests as well as statistical differences for trend were used to compare means for continuously measured variables. $\chi^{2}$ Tests for independence were used to test statistical differences for categorical variables. Data are presented as percentage and mean $\pm \mathrm{SE}$.

\section{Discussion}

In the present study, we found a high prevalence of EI (13.7\%) in patients admitted to the ED. These relevant data are in agreement with a previous observation demonstrating a prevalence of more than $10 \%$ as far as dysnatremia and dyskalemia are concerned [5]. In addition, we also observed crucial difference in EI as related to life decades. In fact, in the E group, we found that EI had the highest prevalence, $22 \%$, whereas it was $11.5 \%$ in the MA group and only $7.1 \%$ in the $Y$ group.

These important data suggest an involvement of aging in determining the different pattern of EI across different decades of life. In this regard, it has been recently reported that the prevalence of hyponatremia and hypokalemia varies with the age [5]. These authors reported that although serum sodium concentration inversely correlated with age, serum potassium, by contrast, did the opposite [5].

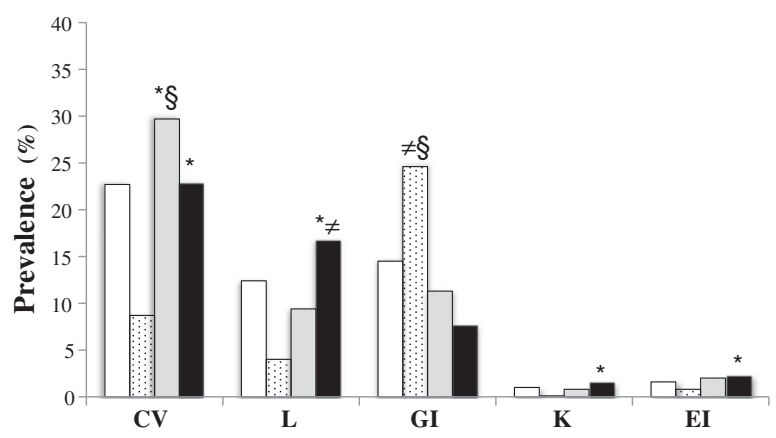

Fig. 2. Diseases associated with EI: cardiovascular disease, lung disease, GI disease, kidney diseases, and EI alone in all patients group (white column), Y group (dotted column), MA group (gray column), and E group (black column). Values are expressed as percentages. ${ }^{*} P<.05$ vs $Y$ group; ${ }^{\neq} P<.05$ vs MA group; ${ }^{\S} P<0.05$ vs $E$ group.
Moreover, Tareen et al [10] have reported that the prevalence of hyponatremia ranges from $2.5 \%$ in younger patients to $50 \%$ in the elderly population. In our study, in particular, hyponatremia occurred in $47.7 \%$ of the E group, in $39.8 \%$ in the MA group, and only in $33.4 \%$ of the $Y$ group (Fig. 1). Similarly, Lindner et al [5] recently showed that the prevalence of hyponatremia in the ED was increased with age up to $15.2 \%$ and $16.9 \%$ in patients older than 71 and 80 years, respectively.

Of interest, we also found that hyponatremia was the most frequent EI in the ED only in the E group, whereas hypokalemia appeared to be the most frequent $\mathrm{EI}$ in the MA and Y groups (48\% and 58.7\%, respectively). This age-related trend of hypokalemia is in agreement with data reported by Lindner et al [5], who previously showed that the prevalence of hypokalemia was $12.8 \%$ in patients between 21 and 30 years old, $11.4 \%$ in patients between 51 and 60 years old, and $10.9 \%$ in patients between 71 and 80 years old.

The reason for such differences remains circumstantial. However, it has been postulated that a loss in kidney function in parallel with aging may plays a crucial role in the age-related derangement of electrolyte metabolism shown in patients admitted to ED [5]. In this regard, we observed, as expected, a significant age-related decline in renal function in parallel with aging (Table 1). As a matter of fact, the physiological age-related decline in renal function, as demonstrated by Lindeman et al [11], would be expected to be approximately $0.75 \mathrm{~mL} / \mathrm{min}$ per year. Thus, it cannot be excluded that the physiological age-related decline in renal function could affect at least in part sodium balance through either volume expansion or distal tubule dysfunction, thus inducing hyponatremia. Similarly, an age-related decline in renal function may cause hyperkalemia through tubule dysfunction; we also observed a rising prevalence of hyperkalemia with age in the ED. In fact, we found that the prevalence of hyperkalemia was $2.4 \%$ and $7.4 \%$ in the Y and MA groups, respectively, whereas it rose up to $19.5 \%$ in the E group. This pattern goes in parallel with the decline of renal function.

As to EI without associated diseases, we found a negligible prevalence of EI (1.6\%). By contrast, we observed that EI was more frequently associated in the Y group with GI diseases (24.6\% of all EI); in the MA group, with cardiovascular diseases (29.7\% of all $\mathrm{EI}$ ); and in the $\mathrm{E}$ group, with both cardiovascular and lung diseases ( $22.8 \%$ and $16.7 \%$, respectively) (Fig. 2).

These data could also explain the high prevalence of hypokalemia observed in the Y group because GI diseases are often associated with hypokalemia. In fact, the prevalence of hypokalemia was reduced in the MA and E groups, who were characterized by only $11.3 \%$ and $7.7 \%$ of GI diseases, respectively (Fig. 2). The reason for such differences could be that young patients may be mainly affect by vomiting and diarrhea that could account for the higher prevalence of hypokalemia. In this regard, we also find a significant inverse correlation in all patients between potassium levels and a prevalence of GI diseases $(r=-0.66)$.

It is not surprising to find that MA and E groups were mainly characterized by EI associated with cardiovascular diseases. In fact, these patients are more frequently treated with drugs somehow affecting electrolyte metabolism (diuretics, angiotensin-converting enzyme inhibitors, angiotensin II receptor blockers, etc). Our data also demonstrated that, in the E group, at variance with the MA group, EI was mainly associated with both cardiovascular and lung diseases. These associations may underline the importance of lung diseases in addition to the cardiovascular diseases in the prevalence of EI in the elderly.

In conclusion, we reported that the prevalence of EI was $13.7 \%$ of all patients admitted to ED and increased to $22 \%$ in the elderly. In addition, the prevalence of EI alone at the ED was only in $1.6 \%$ of cases. So, most patients showing EI are affected by other systemic diseases. As to age influence, EI was more frequently associated in the Y group with GI disease (24.6\%); in the MA group, with cardiovascular disease (29.7\%); and in the E group, with both cardiovascular and lung diseases (22.8\% and $16.7 \%$, respectively). Therefore, the burden of comorbidity as well as aging seems to influence the complex scenario of electrolyte metabolism derangement in ED. Thus, present data suggest that ED physicians 
need to be aware of the potential electrolyte abnormalities that can arise on patient age and its pathologic conditions associated to prevent adverse outcomes.

\section{References}

[1] Arampatzis S, Funk GC, Leichtle AB, Fiedler GM, Schwarz C, Zimmermann H, et al. Impact of diuretic therapy-associated electrolyte disorders present on admission to the emergency department: a cross-sectional analysis. BMC Med 2013;11:83-9.

[2] Funk GC, Lindner G, Druml W, Metnitz B, Schwarz C, Bauer P, et al. Incidence and prognosis of dysnatremias present on ICU admission. Intensive Care Med 2010; 36(2):304-11.

[3] Corona G, Giuliani C, Parenti G, Norello D, Verbalis JG, Forti G, et al. Moderate hyponatremia is associated with increased risk of mortality: evidence from a meta-analysis. PLoS One 2013;8(12):e80451-62.

[4] Balci AK, Koksal O, Kose A, Armagan E, Ozdemir F, Inal T, et al. General characteristics of patients with electrolyte imbalance admitted to emergency department. World J Emerg Med 2013;4(2):113-6.
[5] Lindner G, Pfortmüller CA, Leichtle AB, Fiedler GM, Exadaktylos AK. Age-related variety in electrolyte levels and prevalence of dysnatremias and dyskalemias in patients presenting to the emergency department. Gerontology 2014;60(5):420-3 [Epub 2014 May 20].

[6] Marti G, Schwarz C, Leichtle AB, Fiedler GM, Arampatzis S, Exadaktylos AK, et al. Etiology and symptoms of severe hypokalemia in emergency department patients. Eur J Emerg Med 2014;21(1):46-51.

[7] Najeeb Q, Ruqaya A, Sajad H, Sabiya M, Ruhi A. Electrolyte abnormalities in patients admitted in emergency department of tertiary care institute: a cross sectional study. Int J Med Sci Public Health 2014;3(11):1368-71.

[8] Lee CT, Guo HR, Chen JB. Hyponatremia in the emergency department. Am J Emerg Med 2000;18(3):264-8.

[9] Levey AS, Stevens LA. Estimating GFR using the CKD epidemiology collaboration (CKD-EPI) creatinine equation: more accurate GFR estimates, lower CKD prevalence estimates, and better risk predictions. Am J Kidney Dis 2010; 55(4):622-7.

[10] Tareen N, Martins D, Nagami G, Levine B, Norris KC. Sodium disorders in the elderly. J Natl Med Assoc 2005;97:217-24.

[11] Lindeman RD, Tobin J, Shock NW. Longitudinal studies on the rate of decline in renal function with age. J Am Geriatr Soc 1985;33(4):278-85. 